\title{
THE DEVELOPMENT AND APPLICATION OF TONGUE DIAGNOSIS CABIN
}

\author{
Yu-Hui $\mathrm{Ma}^{1}$, Lin-Feng $\mathrm{Li}^{1}$, Yu-Xuan Zhang ${ }^{2}$, Yu Zhang ${ }^{3}$, Yu-ting Wang ${ }^{4}$, Si-yuan Han ${ }^{2}$, Yu-Rong \\ Liang*1 \\ ${ }^{1}$ College of Chinese Medicine, Hebei University, Baoding, Hebei Province, China. \\ ${ }^{2}$ College of Cyberspace Security and Computer Science, Hebei University, Baoding, Hebei Province, \\ China. \\ ${ }^{3}$ College of Medicine, Hebei University, Baoding, Hebei Province, China. \\ ${ }^{4}$ College of Management, Hebei University, Baoding, Hebei Province, China.
}

Article Info: Received 11 March 2019; Accepted 14 April. 2019

Cite this article as: Ma, Y.-, Li, L.-, Zhang, Y.-, Zhang, Y., Wang, Y.-, Han, S.-, \& Liang, Y.-. (2019). THE DEVELOPMENT AND APPLICATION OF TONGUE DIAGNOSIS CABIN. Journal of Biomedical and Pharmaceutical Research, 8(2).

DOI: https://doi.org/10.32553/jbpr.v8i2.587

Address for Correspondence: Yu-Rong Liang, College of Chinese Medicine, Hebei University, Baoding, Hebei Province, China.

Conflict of interest statement: No conflict of interest

\section{ABSTRACT:}

On the basis of a large number of previous studies, we develop the TCM Tongue diagnosis mobile phone software based on artificial intelligence technology which is named Tongue Diagnosis Cabin. The software utilizes and improves the digital objectification technology of modern tongue image to overcome the influence of the pixel limitation of the mobile phone, the complex shooting environment, the unstable light source and other conditions on the color and definition of the original tongue image, and reduces the total difference between the remote tongue image and the original tongue image color, so as to achieve clinical visual equivalence. The software is based on the mobile phone, which is easy to operate with intelligently guidance and superior performance, and can promote the development of digitalization and telemedicine of tongue diagnosis.

Keywords: tongue diagnosis cabin; tongue diagnosis; intelligence; mobile; telemedicine

\section{INTRODUCTION}

The main content and means of the diagnostic system of TCM are "looking for information, asking for information and asking for information". Tongue diagnosis is an important part of traditional Chinese medicine diagnosis, which is also an important means widely used in clinical diagnosis of TCM. It is an important diagnostic and therapeutic technology to assist the diagnosis and differentiation of diseases by observing the changes of tongue color, shape and fur, which provides important information for the diagnosis of diseases. At present, TCM telemedicine services are developing rapidly. However, when most patients use mobile phones to take tongue pictures, they often cause great interference to the remote diagnosis and treatment of traditional Chinese medicine because of their irregular photographic mode, unclear image and large color error, which even causes misdiagnosis. 
With the strong support of the state for Chinese medicine and the rapid development of modern science and technology, digital image processing technology is gradually applied in the field of Chinese medicine diagnosis, especially for the field of tongue diagnosis of traditional Chinese medicine. Since the 20th century, many scholars and colleges have conducted in-depth research on the digital camera technology of tongue diagnosis in traditional Chinese medicine.The research of digital camera technology mainly includes two aspects: tongue image information collection and tongue image analysis. In the collection of tongue image information, Gao Kai ${ }^{[1]}$ used LED light-emitting diodes as light source and integrating sphere as lighting conditions, he used digital cameras to collect tongue images, which reduced the objective influence of light source and illumination uniformity on tongue image collection; Shanghai University of Traditional Chinese Medicine ${ }^{[2]}$ adopts the technology of high-frequency fluorescence constant light source system, which makes the collection environment more stable and the image color reducibility and truthfulness are higher. In terms of tongue image analysis, Shanghai Jiaotong University ${ }^{[3]}$ used statistical methods to calibrate tongue texture and tongue coating points to determine the algorithm for calculating the tongue color; Zhang Yongtao et al. ${ }^{[4]}$ used the tongue color Lab value to analyze teeth-printed tongue, the result of which is the tongue color $a, b$ values of the tongue map are significantly lower than other tongue colors, so as to make an effective analysis of the teeth-printed tongue; Chen Songhe et al. ${ }^{[5]}$ use CIELAB and LCH color space model to scan and analyze the color space, which ensured the real reproduction of tongue color data in the process of transmission and processing. From above all, we can see that the research of digital tongue image technology is becoming more and more mature, and with the development of science and technology, the intelligent collection and analysis of digital tongue image has become a hot research in the direction of artificial intelligence technology.

At present, there are many tongue picture instruments that have been successfully developed and put into use in the market, which meets the needs of the objective digitization of traditional Chinese medicine tongue diagnosis to some degree, such as the products represented by Xinman's SX-III tongue imager which guarantees the authenticity of the tongue image and the analysis effect has higher accuracy, but because of its high price, the submission is cumbersome, and has great limitations in the use of space and time, it is difficult to be widely used by ordinary families. Based on this, we put forward the concept of "tongue diagnosis cabin"-tongue digitalization software, and inherit the advantages of existing instrument related image processing, with mobile phone image shooting, it gets rid of valuable equipment constraints. On the basis of previous research, we developed a mobile terminal software for tongue diagnosis of traditional Chinese medicine based on artificial intelligence technology- "Tongue Diagnosis Cabin".

\section{DESIGN IDEAS OF "TONGUE DIAGNOSIS CABIN"}

Tongue Diagnosis Cabin adopts the common solution in the field of tongue image digitization. The intelligent voice guides the patients to take correct photos by using the camera of the mobile phone under the proper illumination environment, and then automatically restores the captured tongue image, and a clinically visually equivalent tongue image will be generated for the user in 15 seconds, and carries out automatic analysis of tongue image to form the reference report of tongue image. The design idea is shown in Figure 1. 


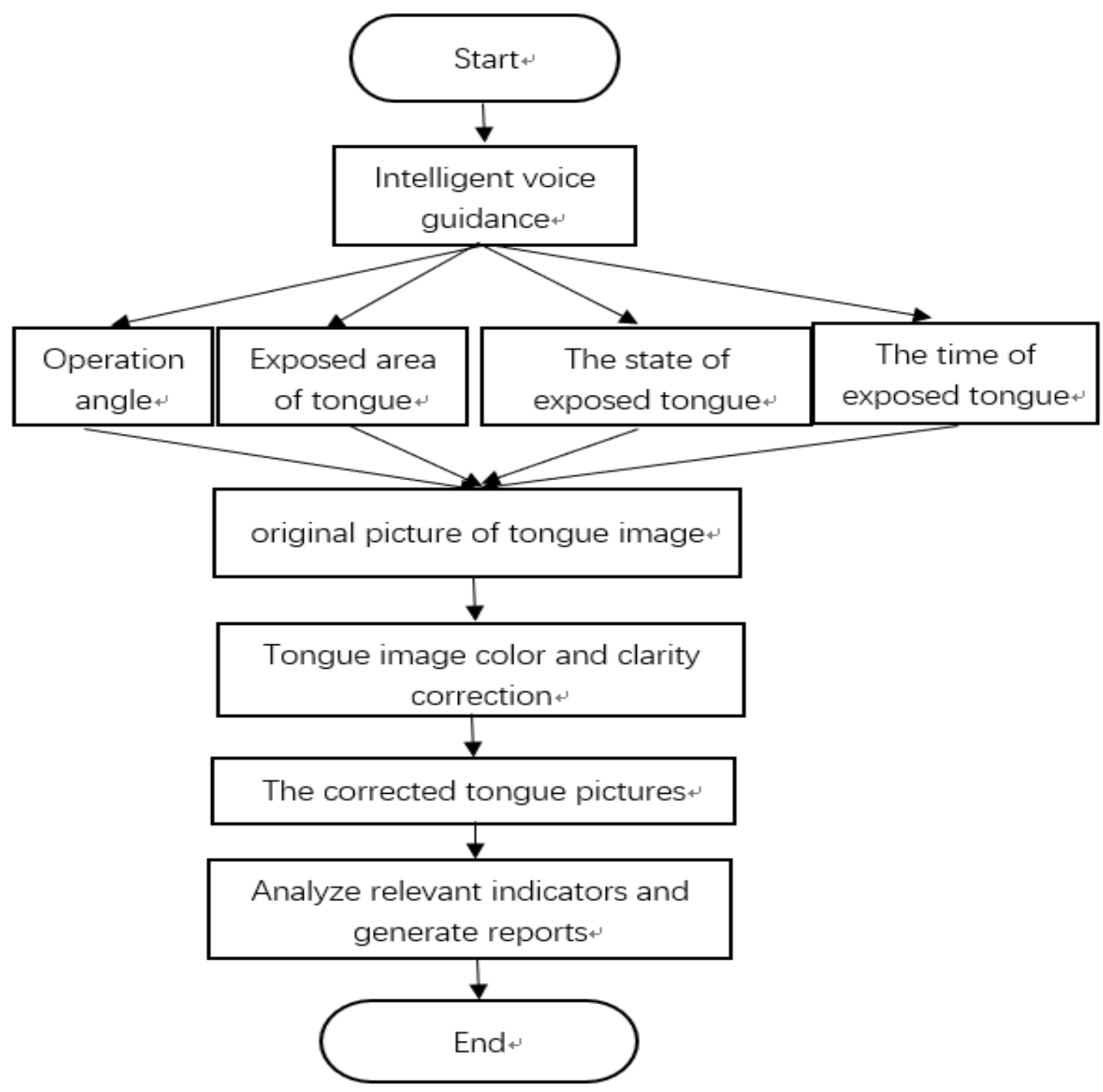

Figure 1: Design schematic diagram of Tongue Diagnosis Cabin

\section{DESIGN OF TONGUE DIAGNOSIS CABIN'S FUNCTION}

\subsection{Intelligent restoration of tongue image to generate clinical equivalent visual photos}

\subsubsection{The recognition of shooting environment}

Due to the advantages of easy operation, fast imaging and easy transmission, most users are accustomed to using mobile phone to capture tongue images and use them for health consultation or telemedicine. However, because of the difference of color temperature, light illumination, shadow and mobile phone model, there are many problems appearing like the image color difference, low resolution and so on, and the tongue image cannot accurately reflect the medical significance of the tongue, causing serious consequences. Tongue Diagnosis Cabin will use the $\mathrm{Al}$ and color analysis techniques to intelligently detect the shooting environment. When the software is running, the user will first be prompted to choose natural and the non-natural light environment. At the same time, the voice suggests that users choose natural light environments as far as possible for tongue imagine acquisition, which may be more standard. After judging the surrounding environment artificially, the software will guide users to the best tongue image collection position in the current environment through voice, and intelligently detect whether the 
relevant environment meets the standard through $\mathrm{Al}$, illumination detection, color temperature detection and other technologies. If the collection standard is met, the software will start to work; if the collection standard is not met, the voice prompts users to adjust the shooting environment or select the natural light for tongue image collection. As shown in Figure 2.

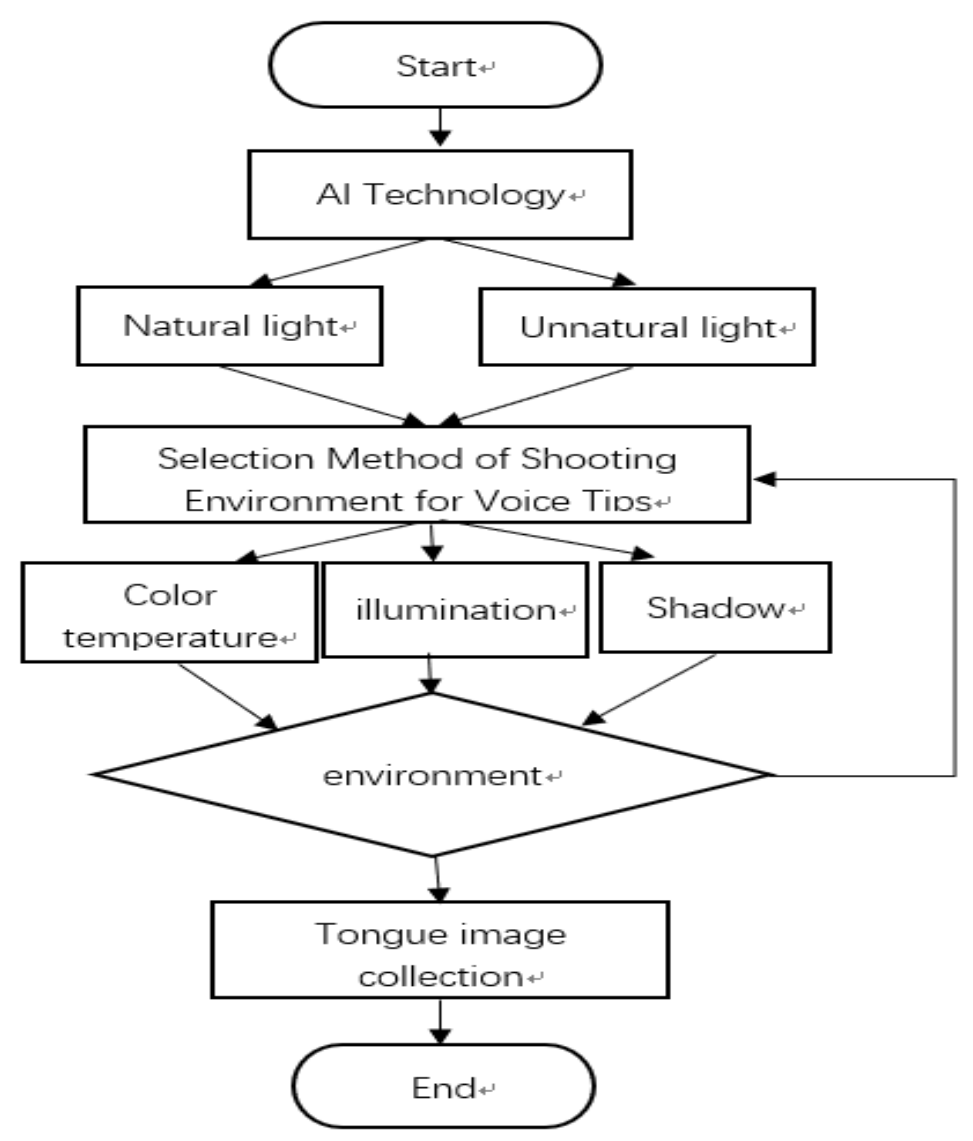

Figure 2: Flow chart of shooting environment identification

\subsubsection{Intelligent voice guidance}

The image error of the tongue image is not only caused by the interference of the shooting environment of the mobile phone, but also related to the factors such as the irregular operation angle, the exposed area of the tongue, the exposed state and the exposed time. Without systematic Chinese medicine education, it is difficult for users to complete all operations correctly, which affects the imaging effect of tongue image and is not conducive to the development of telemedicine services.
In response to the above operational problems, Tongue Diagnosis Cabin uses Al technology and mobile phone sensing system, through voice prompts and human-computer interaction mode, to help users complete the corresponding image acquisition accurately, standardizedly and easily. When entering the tongue image collection function, the software provides users with a standard tongue type collection frame. Through face recognition and other related technologies to determine the tongue area, state, and voice prompts to adjust user acquisition action, tongue exposure 
area, perception of tongue exposure state for tongue image acquisition.

\subsubsection{Automatic restoration of tongue image}

After collecting the picture of the tongue image, Tongue Diagnosis Cabin will analyze and process the picture in the aspects of sharpness, color contrast pixel correction and color reproduction. Through the Interpolation Based, CIE2000, Matlab, Munsell colorful contrast and other techniques, tongue image correction is completed, which improves the clarity to 1.56 times the original pixel and the total color difference is less than 5.0, then the clinical visual equivalent is obtained, thus laying the foundation for automatic analysis of tongue image.

\subsection{Automatic analysis of tongue image to form a reference analysis report of tongue image.}

In the analysis stage, based on the obtained pictures, color and other factors of which meet the requirements, the software further analyzes and studies the tongue image on the tongue shape, tongue color, tongue coating color, putrid, moist, tooth marks and so on. Data cursor mode, marking watershed algorithm, Fourier transform, drying coefficient and other parameter analysis techniques are used in the tongue diagnosis cabin, and the aggregation operation is compared with DS01$\mathrm{B}$ database, so as to realize automatic analysis of tongue image (tongue color, tongue coating, cracks, dental marks, prickles, etc.) characteristic parameters. According to the fixed format of the analysis report, the software will automatically fill in the data of the user's tongue image, and finally generate a PDF image reference analysis report.

\subsection{Long-term follow-up analysis of tongue image to generate electronic medical record of tongue image}

The Tongue Diagnosis Cabin provides file storage for each user's tongue image data, tracks and analyzes relevant data for a long time. Regularly push users' health dynamic reports, provide personal electronic tongue medical record printing service, and provide effective basis for users to state the development of the disease.

\section{CLINICAL APPLICATION OF THE TONGUE DIAGNOSIS CABIN}

Tongue, the seedling of heart and the external conditions of the spleen and stomach, connect with the five Zang-organs and six Fu-organs through the meridians, which can reflect the changes of qi, blood and body fluid in the Zang-Fu organs. As an important part of the diagnosis in TCM, it is important to diagnose the disease and judge the condition ${ }^{[6]}$. Tongue diagnosis is one of the most distinctive diagnosis methods in traditional Chinese medicine diagnosis methods. After thousands of years of inheritance and development, it has become a systematic and comprehensive diagnosis method. Tongue image can reflect the health and disease state of the human body all the time, and it has great significance for the judgment of health status, the diagnosis and prognosis of disease syndrome.

The ultimate goal of the development of tongue image software is to be apply in clinic. Therefore, we popularize the tongue diagnosis cabin to clinic, and classify the collected tongue images, and combine them with the modern Chinese medicine constitution theory system. After comparative analysis, we found that the proportion of teeth-printed tongue and pale tongue in the yang-deficient body is the highest (as shown in Figure 3 and Figure 4).Due to the improvement of living standards in modern society, people like to eat fat and sweet taste, with a long-term drinking, which leads to endoretention of damp heat, and their tongues are red, and tongue coating is yellow and greasy (Figure 5).In addition, we also collected images of the tongue image of patients with coronary heart disease, and 
compared with the results of coronary angiography, found that the plaque tongue

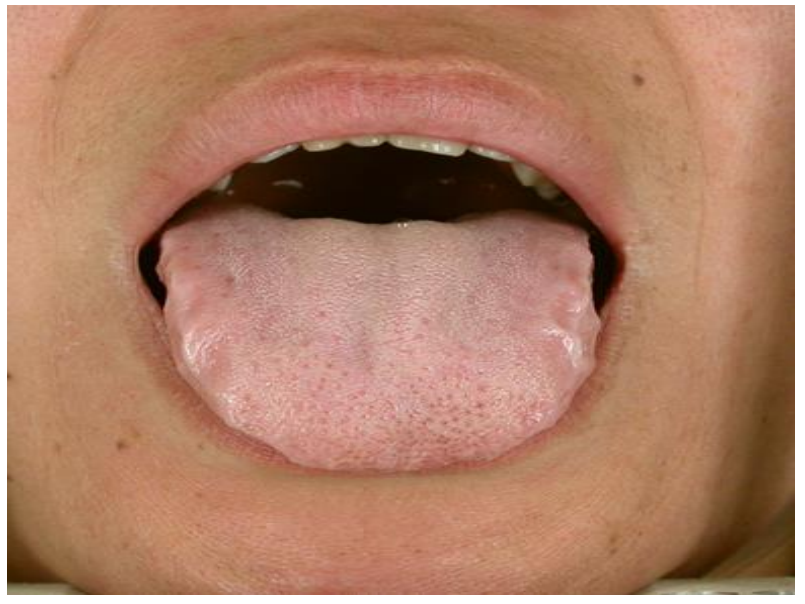

Figure 3 dentate tongue

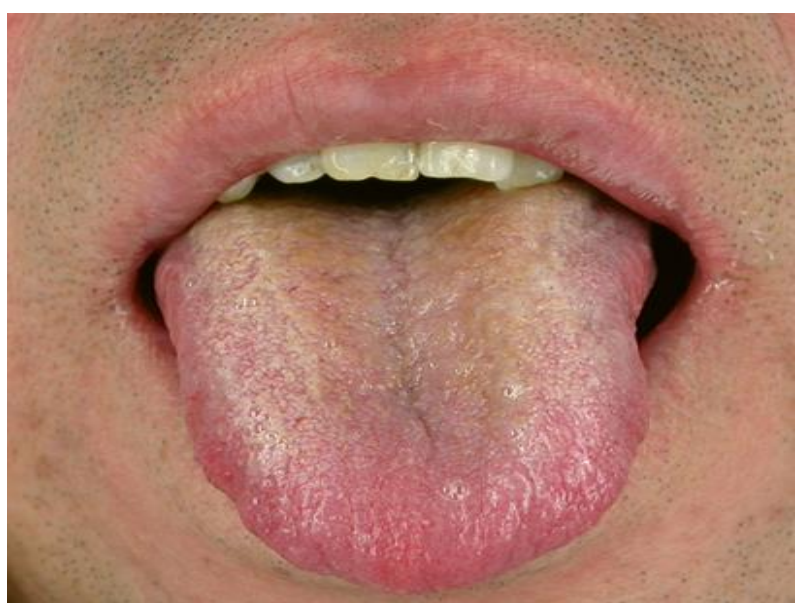

Figure 5 yellow tongue red greasy

\section{SUMMARY}

In recent years, the State Administration of Traditional Chinese Medicine has issued a series of documents ${ }^{[7]}$, pointing out that it is necessary to guide and encourage Chinese medicine hospitals to use information technology and intelligent technology to provide telemedicine, "four diagnoses" of Chinese medicine and macro-and microtongue diagnosis services to lower hospitals and grass-roots medical and health institutions, vigorously develop TCM telemedicine services and improve the accessibility of high-quality TCM medical and the coronary artery obstruction have a certain correlation (Figure 6).

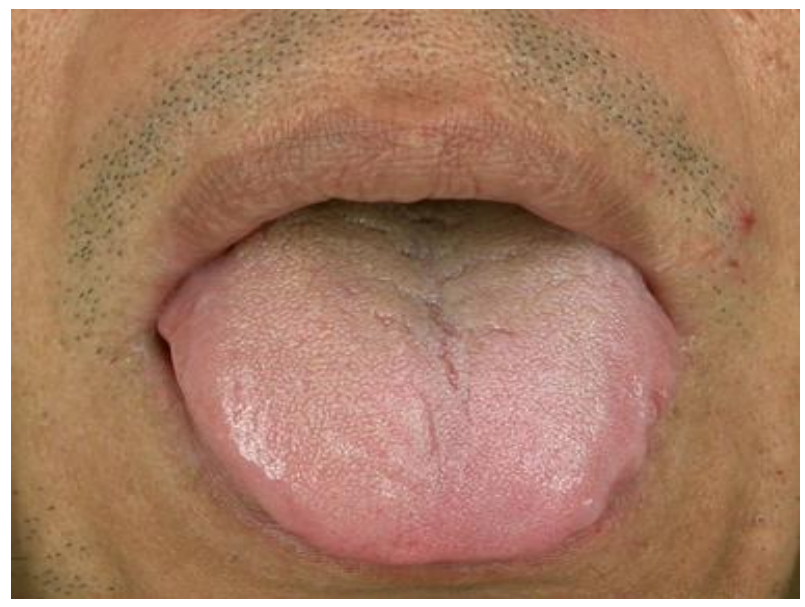

Figure 4 pale tongue

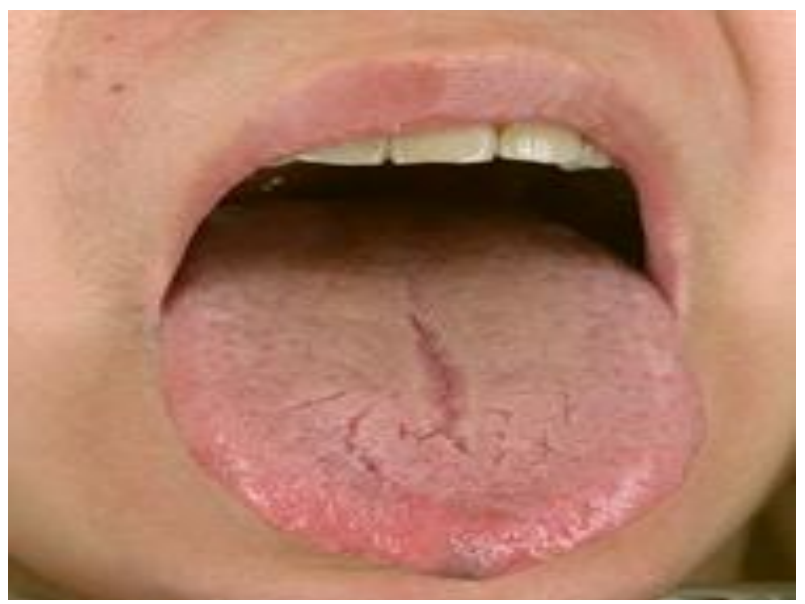

Figure 6 Ecchymosis tongue

resources and overall service. As a mobile terminal tongue diagnosis software, Tongue Diagnosis Cabin combines telemedicine with traditional Chinese medicine tongue diagnosis and construct a complete and credible TCM tongue diagnosis data mining system, which integrates and optimizes modern digital diagnosis and treatment technology, and gets rid of valuable equipment. So it is portable and easy to use, and service the public more extensively.At the same time, it will be better to help doctors diagnose and treat, improve doctors' work efficiency. Rate and effect of diagnosis and treatment can promote the 
development of telemedicine. In the future, it can also be extended to community, medical product research and development company, medical research center and other institutions to collect and integrate tongue image data, so as to provide true and reliable objective image data for the diagnosis and treatment research and product development of different diseases (especially the world's difficult and chronic diseases), and promote the standardization of traditional Chinese medicine tongue diagnosis. And it can promote the revitalization of traditional Chinese medicine culture and promote the innovation and development of traditional Chinese medicine.

\section{REFERENCES}

1. Gao Kai, Bi Ping. Application of image processing technology in the study of tongue image of traditional Chinese medicine [j]. Medical and Health Equipment, 2008 (05): 30-32+38.

2. Lu Chunyan, Xu Jiazhen, Tu Liping. Clinical research and application progress of tongue image diagnosis technology [j]. Shanghai Journal of Traditional Chinese Medicine, 2013, 47 (05): 98-101.
3. Wang Yonggang, Yang Jie, Zhou Yue, et al. Research on color recognition of tongue images in traditional Chinese medicine [j]. Journal of Biomedical Engineering, 2005 (06): 1116-1120.

4. Zhang Yongtao. Analysis of digital tongue map analysis and application on scallop tongue [d]. Beijing University of Chinese Medicine, 2005.

5. Chen Songhe. Research on tongue color analysis method of digital tongue map [d]. Beijing University of Chinese Medicine, 2007.

6. Zhang Ji, Qian Jun, Wang Ruijuan,et al. Analysis of the status quo of objective characteristics analysis of digital tongue images of traditional Chinese medicine [j]. World Science and TechnologyModernization of Traditional Chinese Medicine, 2014, 16(11): 2420-2424.

7. China's first white paper on the development of Chinese medicine, "Chinese Medicine in China" [j]. Journal of Traditional Chinese Medicine, 2017, 58 (02): 95. 